\title{
ANALYSE ÉLÉMENTAIRE DES MATÉRIAUX SANS ÉTALONNAGE
}



Jörg HERMANN ${ }^{1, *}$, Chao SHEN ${ }^{1}$, Antonio HERMANN ${ }^{1}$, Olivier ALEIXO DA LUZ ${ }^{1}$, Aya TALEB ${ }^{1,2}$, Frédéric PELASCINI ${ }^{2}$

${ }^{1}$ Aix-Marseille Université, CNRS, Laboratoire LP3, Marseille, France

${ }^{2}$ Cetim Grand Est, Illkirch-Graffenstaden, France

*hermann@lp3.univ-mrs.fr

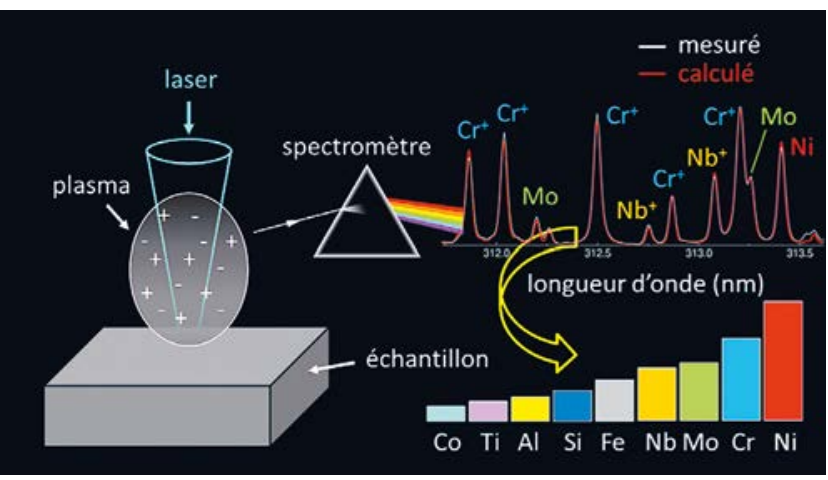

La mesure de la composition élémentaire d'un matériau nécessite habituellement un étalonnage à l'aide de références de composition proche du matériau à analyser. Cela implique de connaître la composition du matériau et d'avoir à disposition des étalons adéquats. La spectroscopie du plasma induit par laser permet de s'affranchir de cette étape d'étalonnage et de réaliser une analyse basée sur la comparaison d'un spectre mesuré avec le spectre calculé à l'aide d'un modèle : c'est l'approche « calibration-free ».

https://doi.org/10.1051/photon/202010346

Article publié en accès libre sous les conditions définies par la licence Creative Commons Attribution License CC-BY (https://creativecommons.org/licenses/by/4.0), qui autorise sans restrictions l'utilisation, la diffusion, et la reproduction sur quelque support que ce soit, sous réserve de citation correcte de la publication originale.

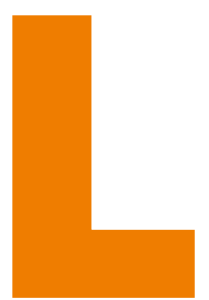

a spectroscopie du plasma induit par laser, connue sous l'acronyme LIBS, a suscité l'intérêt tout d'abord par sa capacité à permettre

l'analyse élémentaire des matériaux sans contact et sans préparation de l'échantillon [1]. Cette technique possède cependant un autre atout qui la rend unique parmi les techniques d'analyse : elle permet de mesurer la composition élémentaire d'un matériau sans nécessité d'un étalonnage préalable. Cela est possible grâce aux propriétés du plasma produit par ablation laser qui permettent de simuler le spectre d'émission à l'aide d'un modèle simple et robuste. Tout d'abord, l'irradiation du matériau par des impulsions laser de courte durée (quelques nanosecondes ou moins) avec une fluence laser de l'ordre de $100 \mathrm{~J} / \mathrm{cm}^{2}$ assure un transfert stœchiométrique de la matière du solide vers le plasma. L'ablation est alors un processus hors équilibre, bien différent du phénomène d'évaporation à l'équilibre thermodynamique que l'on peut observer lors de l'irradiation laser à faible fluence. Lorsque la durée d'impulsion laser est supérieure au temps de thermalisation

Figure 1.

Spectre d'émission du plasma produit par ablation laser d'une pièce de 20 centimes d'Euro. La fenêtre spectrale montre de nombreuses raies émises par les atomes d'éléments majeurs de l'alliage qui sont le cuivre ( $89 \%$ ), l'aluminium (5\%), le zinc (5\%) et de l'étain (1\%). électron-réseau, la matière est vaporisée pendant l'irradiation. Le rayonnement laser chauffe la vapeur en expansion et augmente ainsi ses degrés d'atomisation et d'excitation. En raison de la densité élevée du panache d'ablation, les processus collisionnels dominent les processus radiatifs et ce plasma atteint l'état d'équilibre thermodynamique local très rapidement après l'impulsion laser. Le panache d'ablation subit une expansion rapide pendant quelques dizaines de nanosecondes jusqu'à ce que la vapeur soit en équilibre de pression avec le gaz ambiant. Par la

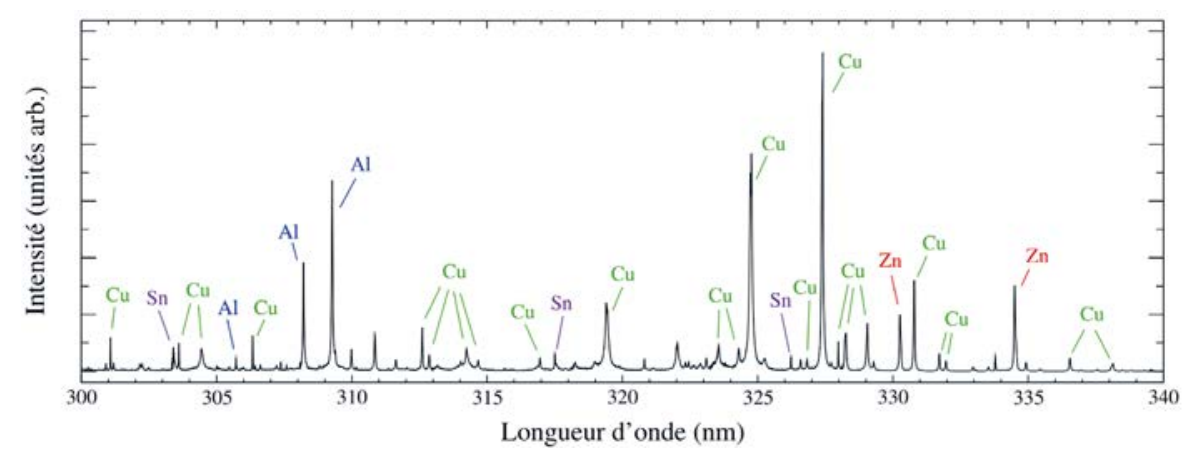


suite, l'expansion se poursuit plus lentement par des processus de diffusion de chaleur et de particules. Lorsque l'ablation laser est généré sous atmosphère d'un gaz inerte tel que l'argon, l'échange d'énergie entre le panache d'ablation et le gaz environnant est minimisé. Le plasma est alors plus brillant que celui produit dans l'air et possède une durée d'émission plus longue. De plus, il est caractérisé par une distribution spatiale uniforme de la température et des densités de ses composants.

Un plasma spatialement uniforme et en équilibre thermodynamique local peut être considéré comme une source de rayonnement idéal, car son spectre d'émission peut être calculé aisément et avec précision. Habituellement, les deux propriétés, l'uniformité spatiale et l'état d'équilibre, sont difficiles à obtenir en même temps, car le temps de thermalisation et les temps caractéristiques de diffusion sont similaires pour des plasmas atmosphériques. Le plasma d'ablation laser fait exception à cette règle en raison de sa densité initiale très élevée. En effet, lorsque la densité augmente, la thermalisation est plus rapide tandis que les processus de diffusion sont plus lents [2].

\section{ILLUSTRATION DE LA MÉTHODE PAR L'ANALYSE \\ DES PIËCES DE MONNAIE}

Pour illustrer l'analyse élémentaire sans étalonnage, nous présentons des résultats d'une étude de la composition de pièces de monnaie de 20 centimes d'Euro. Elles sont faites en alliage de type laiton appelé « or nordique ». Plusieurs pièces fabriquées dans différents pays et à différentes dates ont été sélectionnées afin de contrôler s'il existe des différences dans leur composition. Les échantillons sont irradiés par des impulsions laser ultraviolettes d'une durée de 4 ns, délivrée par une source laser Nd:YAG (Quantel, modèle Brilliant). Les irradiations sont faites sous argon afin d'obtenir un plasma approprié pour un calcul simple et précis du spectre d'émission. Le spectre d'émission du plasma est enregistré à l'aide d'un spectromètre à échelle (LTB, modèle Aryelle Butterfly) qui permet d'observer une large fenêtre spectrale avec un pouvoir de résolution spectral élevé. Le spectromètre est équipé d'un détecteur CCD avec intensificateur d'image à porte temporelle. Le choix du délai d'observation par rapport à l'impulsion laser est guidé par deux critères : (i) la validité

\section{ANALYSE PAR MODÉLISATION DU SPECTRE}

À l'équilibre thermodynamique local, l'état du plasma est décrit de façon simplifiée par les lois statistiques d'équilibre. Un plasma composé de néléments dépend alors de $n+1$ paramètres: la température et les densités atomiques des $n$ éléments. Pour des raisons pratiques, nous utilisons un jeu de $n+1$ paramètres équivalents: la température $T$, la densité électronique $n_{e}$ et les fractions de $n$-1 éléments. Le calcul du spectre d'émission du plasma nécessite un paramètre supplémentaire qui est le diamètre $L$ du plasma le long de l'axe optique d'observation. La luminance spectrale du plasma uniforme en ETL est donnée par [3]:

$$
B_{\lambda}=B_{\lambda}^{0}\left(1-\mathrm{e}^{-\alpha L}\right),
$$

où $B_{\lambda}^{0}$ est la luminance spectrale du corps noir et $\alpha$ le coefficient d'émission. Les $n+2$ paramètres sont déduits de la comparaison du spectre ainsi calculé au spectre mesuré en utilisant une procédure itérative : dans une boucle principale, les paramètres sont obtenus successivement et en utilisant une boucle secondaire de comparaison entre spectres calculé et mesuré pour chaque paramètre. L'analyse est terminée lorsque les changements de tous les paramètres sont négligeables par rapport à leurs valeurs absolues.

\section{Laser Optics \\ When Size Matters}

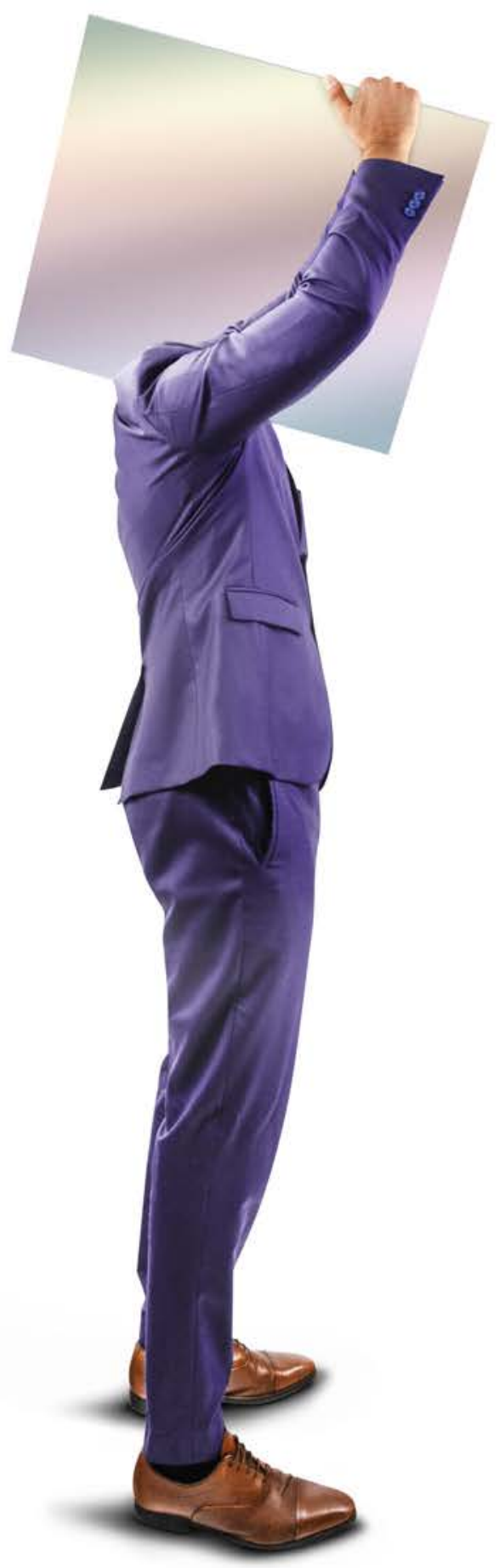

Large custom laser optics with highest damage threshold. 
du modèle et (ii) le rapport signal sur bruit. Le plasma d'ablation laser en expansion est caractérisé par la décroissance de la densité électronique. Il faut donc enregistrer le spectre suffisamment tôt pour que la densité électronique soit assez élevée pour assurer l'état d'équilibre thermodynamique local (ETL). Mais la présence de charges libres génère du rayonnement continu par effets de bremsstrahlung et de recombinaison radiative qui perturbe l'observation de raies spectrales. Le rapport signal sur bruit augmente donc avec le temps et le délai d'observation est choisi le plus grand possible en respectant la condition de l'ETL. La durée de la porte d'observation doit être suffisamment petite pour que les variations de température et de densité électronique au cours de l'observation soient faibles devant leurs valeurs absolues $\left(\Delta T / T, \Delta n_{e} / n_{e}<<1\right)$.

Un spectre, enregistré avec un délai de $2 \mu \mathrm{s}$ par rapport à l'impulsion laser, et une durée de porte de $1 \mu \mathrm{s}$, est présenté sur la figure 1 pour une fenêtre spectrale qui permet d'observer de nombreuses raies émises par des atomes d'éléments majeurs de l'alliage. Le spectre mesuré est comparé à la luminance spectrale du plasma en ETL. Les fractions élémentaires sont déduites du meilleur accord entre le spectre mesuré et celui calculé à l'aide d'une procédure itérative [4].

\section{DIAMÈTRE DU PLASMA MESURÉ À L'AIDE DE L'AUTO-ABSORPTION}

La luminance spectrale du plasma en ETL permet de distinguer deux cas limites selon l'épaisseur optique $\tau=\alpha L$. Pour une raie optiquement mince $(\tau<<1)$, la luminance spectrale (équation 1) est donnée par $B_{\lambda}=\varepsilon_{\lambda} L$. Ici, $\varepsilon_{\lambda}$ est le coefficient d'émission qui est lié au coefficient d'absorption par la loi du rayonnement thermique de Kirchhoff $\varepsilon_{\lambda}=\alpha_{\lambda} B_{\lambda}^{0}$. Pour le cas opposé d'une raie très fortement auto-absorbée ( $\tau \gg>1$ ), la luminance spectrale égalise la valeur du corps noir $B_{\lambda}=B_{\lambda}^{0}$. Dans ce cas, la luminance est indépendante du diamètre $L$, en contraste avec le cas d'une raie optiquement mince dont la luminance augmente linéairement avec $L$. La différence dans la dépendance de $L$ est exploitée pour mesurer le diamètre du plasma à l'aide de deux raies ayant des épaisseurs optiques différentes [5].
La densité électronique est mesurée par élargissement Stark des raies spectrales. Cet effet est illustré sur la figure 2 pour une raie du cuivre. La valeur $n_{e}=4,5 \times 10^{16} \mathrm{~cm}^{-3}$ mesurée pour $t=2,5 \mu$ s est suffisamment élevée pour assurer l'état d'équilibre. Ce délai est donc retenu pour l'analyse, car il présente un bon compromis entre la validité du modèle et un rapport signal sur bruit permettant la mesure d'éléments de trace. La température est déduite du rapport d'intensité entre des raies spectrales provenant des niveaux électroniques avec des énergies différentes. Deux raies sont en principe suffisantes pour mesurer $T$. Cependant, en raison des incertitudes associées aux coefficients

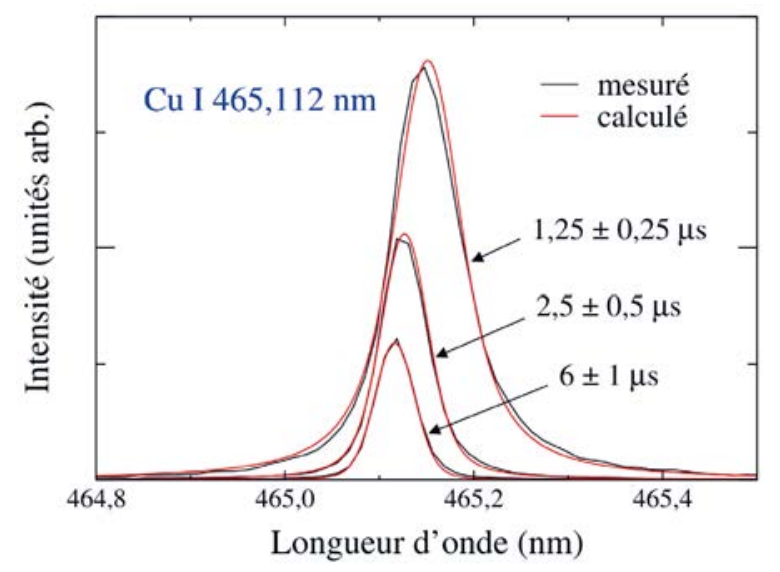

Figure 2.

Profil spectral d'une raie de cuivre observée à différents temps. L'élargissement et le déplacement vers le rouge observés pour les temps tôt sont dus à l'effet Stark et permettent de mesurer la densité électronique.
d'Einstein d'émission spontanée et aux erreurs de mesures d'intensité, la mesure de température se fait souvent à l'aide de multiples raies dont les rapports d'intensité sont présentés sous forme d'un diagramme de Boltzmann [6]. Les fractions élémentaires $C_{x}$ sont ensuite déduites des intensités des raies provenant des atomes ou des ions de tous les éléments.

Les transitions utilisées pour toutes les mesures sont choisies selon les critères suivants : (i) le rapport signal sur bruit suffisamment élevé, (ii) la justesse du coefficient d'Einstein d'émission spontanée, (iii) l'épaisseur optique suffisamment faible. Les plasmas en ETL ayant une densité élevée, l'auto-absorption a une forte influence sur l'intensité d'émission de nombreuses raies. Même si le calcul du spectre théorique tient compte de lauto-absorption, les erreurs de mesure augmentent avec l'épaisseur optique et le choix des «bonnes » raies spectrales est donc crucial pour une analyse robuste. Seule la mesure du diamètre de plasma le long la direction d'observation $L$ nécessite de choisir une raie auto-absorbée.

L'analyse de plusieurs pièces de 20 centimes d'Euro a permis de constater que leur composition est égale en éléments majeurs. Des différences sont observées seulement pour certains éléments mineurs ou des traces, 
comme le montre la variation de l'intensité de raies spectrales sur la figure 3(a). Les plus grandes variations sont observées pour le nickel et le manganèse. Leur abondance dépend faiblement du pays d'origine, mais change significativement avec la date de fabrication (Fig. 3(b)]. À titre d'exemple, la fraction massique du nickel est d'environ $0,1 \%$ pour les pièces fabriquées en 1999 tandis qu'elle est 5 fois plus faible pour celles fabriquées à partir de l'année 2000 [Fig. 3(c)]. Ce changement est attribué aux directives européennes selon lesquelles le nickel présente un danger pour la santé.

\section{CONCLUSION}

En choisissant soigneusement les conditions expérimentales, l'ablation laser permet de générer un plasma qui combine deux propriétés qui sont habituellement difficiles à obtenir en même temps: l'équilibre thermodynamique local et l'uniformité spatiale. La combinaison des deux propriétés caractérise une source de rayonnement idéale dont le spectre d'émission peut être reproduit précisément à l'aide d'un calcul simple. Cela ouvre la voie à l'analyse élémentaire des matériaux sans la nécessité d'un étalonnage préalable. Illustrées ici pour l'analyse des pièces de monnaie, les mesures sans étalonnage peuvent être appliquées à toute sorte de matériaux, indépendamment de leur composition chimique.


$\mathrm{Cu} \mathrm{Zn} \mathrm{Al} \mathrm{Sn} \mathrm{Ni} \mathrm{Fe} \mathrm{Mn} \mathrm{K} \mathrm{Si} \mathrm{Na} \mathrm{Mg} \mathrm{Ca}$

Figure 3.

(a) Spectres enregistrés pour des pièces de 20 centimes d'Euro fabriquées dans plusieurs pays et à des dates différentes. Les deux zones spectrales illustrent un changement dans l'abondance des traces de nickel et de manganèse. (b) Fraction massique des éléments $C_{\text {massique }}$ déduites de l'analyse LIBS. (c) Ratio entre fractions des pièces fabriquées en 1999 et celles des pièces fabriquées après 1999.

\section{REMERCIEMENTS}

J. H. tient à remercier Bernard Fontaine pour ses conseils scientifiques et rédactionnels.

\section{UN BOND \\ DANS LE NANO \\ POSITIONNEMENT \\ PAR SYSTÈME PIEZOÉleCTRIQUE -}

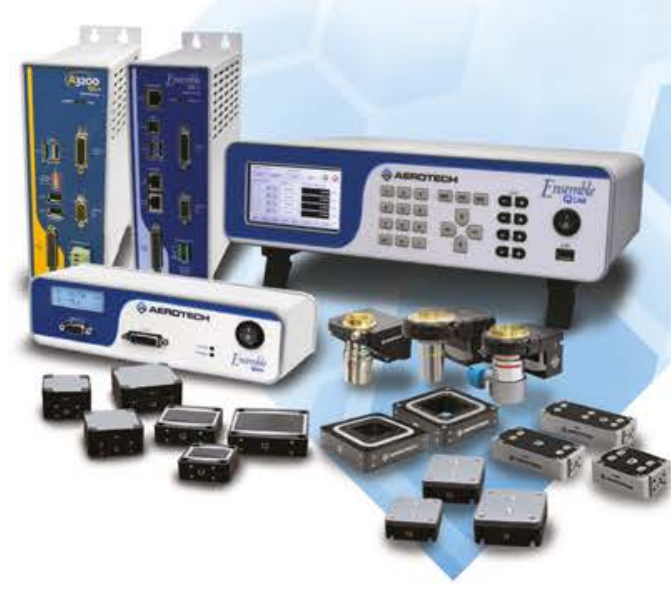

\section{Lancement de la série: nano-positionneur piezo électrique QNP et Piezo contrôleur QLAB}

Les tables QNP présentent une raideur hors norme grâce à une fréquence de résonnance très élevée et une résolution sub-nanométrique. Elles sont donc idéales pour les applications pointues à faible encombrement telles que l'intérferometrie, la microscopie et les alignements d'extrême précision.

Le contrôleur associé QLAB dispose d'un écran tactile et peut fonctionner de manière indépendante ou peut être connecté à un PC via Ethernet, ce qui le rend extrémement flexible dans toutes les situations. Avec des performances sub-nanométriques et un environnement de contrôle et de programmation très convivial, obtenir un positionnement nanométrique n'aura jamais été aussi facile.

\section{RÉFÉRENCES}

\section{[1] D. W. Hahn, N. Omenetto, Appl. Spectrosc. 66, 347 (2012)}

[2] J. Hermann, D. Grojo, E. Axente et al., Phys. Rev. E 96, 053210 (2017)

[3] J. Cooper, Rep. Prog. Phys. 29, 35 (1966)

[4] J. Hermann, System and method for quantitative analysis of the elemental composition of a material by laser-induced breakdown spectroscopy (LIBS), brevet US8942927B2 (2015)

[5] C. Gerhard, J. Hermann, L. Mercadier et al., Spectrochim. Acta Part B 101, 32 (2014)

[6] J. Hermann, E. Axente, F. Pelascini, V. Craciun, Anal. Chem. 91, 2544 (2019) 